\title{
Relationship between Electronic and Geometric Structures of the $\mathrm{O} / \mathrm{Cu}(001)$ System
}

\author{
Sergey Stolbov and Talat S. Rahman \\ Department of Physics, Cardwell Hall, \\ Kansas State University, Manhattan, KS 66506
}

\begin{abstract}
The electronic structure of the $(2 \sqrt{2} \times \sqrt{2}) R 45^{\circ} \mathrm{O} / \mathrm{Cu}(001)$ system has been calculated using locally self-consistent, real space multiple scattering technique based on first principles. Oxygen atoms are found to perturb differentially the long-range Madelung potentials, and hence the local electronic subbands at neighboring $\mathrm{Cu}$ sites. As a result the hybridization of the oxygen electronic states with those of its neighbors leads to bonding of varying ionic and covalent mix. Comparison of results with those for the $c(2 \times 2)$ overlayer shows that the perturbation is much stronger and the Coulomb lattice energy much higher for it than for the $(2 \sqrt{2} \times \sqrt{2}) R 45^{\circ}$ phase. The main driving force for the $0.5 \mathrm{ML}$ oxygen surface structure formation on $\mathrm{Cu}(001)$ is thus the long-range Coulomb interaction which also controls the charge transfer and chemical binding in the system.
\end{abstract}

PACS numbers: 68.43, 73.20.Hb, 71.15.Ap

\section{INTRODUCTION}

Experimental structural studies of $\mathrm{Cu}(001)$ with sub-monolayer coverage of oxygen shows clear signs of phase instability of the system (see for example Ref. 1). The recent studies are a continuation of the debate that has circled around two main phases: a $c(2 \times 2)$ structure on an unreconstructed $\mathrm{Cu}(001)$ 目胜国 and a $(2 \sqrt{2} \times \sqrt{2}) R 45^{\circ}$ structure which involves a reconstructed surfacel. Some experiments have indicated the presence of both phases Still other have affirmed the presence of the $c(2 \times 2)$ overlayer at low coverages, but only in nanometer size domains and not in large well-ordered areas 9 . Based on these experimental results, it is now widely accepted that for coverage lower than $0.34 \mathrm{ML}$, oxygen forms $c(2 \times 2)$ islands. If the coverage exceeds this value, the $(2 \sqrt{2} \times \sqrt{2}) R 45^{\circ}$ structure is formedi. An array of experimental measurements based on surface X-ray diffraction 10 , low energy elec- 
tron diffraction 1 月, 2 , surface extended X-ray adsorption provided useful, and at times conflicting, information on $(2 \sqrt{2} \times \sqrt{2}) R 45^{\circ}$ superstructure of $\mathrm{O}$ on $\mathrm{Cu}(001)$. One of the common features of the superstructure, schematically illustrated in Fig. 1, from these studies is the missing of every fourth (010) row of atoms from the top $\mathrm{Cu}$ layer. Secondly, all studies point to the oxygen atoms occupying sites located on either side of the missing row between $\mathrm{Cu}$ atoms (denoted as $\mathrm{Cu} 2$ in Fig. 1). The structure undergoes further reconstruction (some displacement of $\mathrm{Cu} 2$ along the (100) direction) and relaxation of mostly the top $\mathrm{Cu}$ layer. There is, however, some discrepancy in the exact nature of the relaxation obtained from different experiments. For example, according to Ref. 12 the heights $z_{O}, z_{C u 1}$ and $z_{C u 2}$ in Fig. 1, appear in the order $z_{O}>z_{C u 2}>z_{C u 1}$, whereas Ref. 目 gives $z_{C u 1}>z_{O}>z_{C u 2}$ (see Table \for details).

The competition between the two observed phases of $\mathrm{O}$ on $\mathrm{Cu}(001)$ and the subsequent reconstruction of the surface with the $(2 \sqrt{2} \times \sqrt{2}) R 45^{\circ}$ overlayer naturally beg the question about the origin of this complex behavior. The driving forces for such structural transformation are expected to be related to the chemical binding and electronic structure of the system. Earlier theoretical studies, based on simple models, have correlated the structural properties of $\mathrm{O} / \mathrm{Cu}(001)$ system to a strong $p \mathrm{O}-d \mathrm{Cu}$ hybridization ${ }^{3}$ and charge transfer 14 in the vicinity of the surface. The rationale of these assumptions is supported by calculations, which indicate the presence of the charge transfer from the metal atoms to oxygen 15.16 and a strong $p \mathrm{O}-d \mathrm{Cu}$ hybridization 17 for $\mathrm{O} / \mathrm{Cu}(001) c(2 \times 2)$ system. Our recent first principle calculations for the $c(2 \times 2) \mathrm{O}$ overlayer on $\mathrm{Cu}(001)^{18}$ also confirm the importance of considerations of hybridization and charge transfer in the system and show that these features are eventually traceable to the long-range Coulomb interaction in the system. Clearly, the electronic structure and chemical binding in this system are complex and an adequate description of the relationship between the electronic and atomic structures of $\mathrm{O} / \mathrm{Cu}(001)$ is yet to be established for the $(2 \sqrt{2} \times \sqrt{2}) R 45^{\circ}$ structure. Our goal here is to carry out such a study to understand the driving force for the observed surface reconstruction. For this purpose we take as benchmarks the two proposed surface configurations from Ref. [1 and 12 and compare their implications. As we shall see, with minor differences in the electronic structure, both geometries provide the same rationale for the ensuing missing row reconstruction. Comparison of the results with those for the $c(2 \times 2)$ phase provides further evidence for the relative stability of the $(2 \sqrt{2} \times \sqrt{2}) R 45^{\circ} \mathrm{O}$ overlayer for 0.5 ML coverage. 
The calculations in the present paper have been carried out using the real space multiple scattering technique. We have chosen this particular method because apart from being based on first principles, it allows calculations of the charge transfer in the system with ease and provides a good estimate of the nature of the local bonding. This technique also lends itself nicely to the calculation of local densities of electronic states, their projections on the cubic harmonics, and the valence electronic charge densities. We also calculate the lattice Coulomb energies and the perturbation of the Madelung potential induced by the overlayer.

\section{COMPUTATIONAL DETAILS}

Our calculations are based on the density functional theory within the local density approximation (LDA) ${ }^{19}$ and multiple scattering theory. We use the local self-consistent multiple scattering (LSMS) scheme20, which is designed for extended heterogeneous solids. Within LSMS a compound is divided into overlapping clusters called local interaction zones (LIZs) centered around atoms of different local environments. For each LIZ, we solve a system of equations for the $T$ - scattering matrix in the lattice site - angular momentum representation for the muffin-tin (MT) potentia21:

$$
\sum_{n_{1} L_{1}}\left\{\delta_{L^{\prime} L_{1}}^{n^{\prime} n_{1}} t_{n^{\prime} l^{\prime}}^{-1}-g_{L^{\prime} L_{1}}^{n^{\prime} n_{1}}\right\} T_{L_{1} L}^{n_{1} n}=\delta_{L L^{\prime}}^{n n^{\prime}}
$$

where $n$ denotes the lattice site number, $L=l, m$ are angular momentums, $t_{n l}$ are the single-site scattering matrix elements, $g_{L L_{1}}^{n n_{1}}$ denote the free-electron Green's function matrix elements, and $T_{L L^{\prime}}^{n n^{\prime}}$ are scattering path matrix elements. The solutions are used to determine the LIZ Green's functions, the local densities $N_{l}^{n}(E)$ of electronic states, their projections $N_{l m}^{n}(E)$ on the cubic harmonics, and local valence charge densities for the atoms located at the center of each LIZ. These charge densities are used further to solve the Poisson's equation for the entire extended system and hence to build a new potential for the next iteration of the self-consistent process. An application of this method to materials related

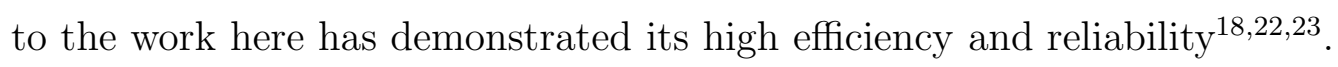

To take into account surface effects on the MT-potential, we follow the approach developed in Ref. 24. In this approach the space in the vicinity of the surface is divided into layers belonging to different atomic planes parallel to the surface and the interstitial charge density $\rho_{i}$ is supposed to be layer-dependent reflecting the asymmetry of the system. The 
detailed description of the technique is given elsewhere 18 . Here we only remind our readers that in our calculations the Madelung potential includes monopole $M_{i \alpha, j \beta}^{00}$, dipole $M_{i \alpha, j \beta}^{10}$ and interstitial $V_{i j}\left[\rho_{j}\right]$ terms 24 :

$$
V_{i \alpha}^{M a d}=\sum_{j, \beta}\left(q_{j \beta} M_{i \alpha, j \beta}^{00}+d_{j \beta} M_{i \alpha, j \beta}^{10}+V_{i j}\left[\rho_{j}\right]\right)
$$

where $i, j$ denote the layer number, while $\alpha, \beta$ number the atoms in the $2 \mathrm{D}$ unit cell, $d_{j \beta}$ is a dipole moment of the MT charge density, and the effective charge

$$
q_{j \beta}=Z_{j \beta}-q_{j \beta}^{M T}+\frac{4 \pi}{3} \rho_{j} R_{j \beta}^{3}
$$

The exchange and correlation parts of the potential are determined within LDA using the technique described in Ref. 25.

In this work the above formalism is applied to the case of $(2 \sqrt{2} \times \sqrt{2}) R 45^{\circ} \mathrm{O}$ overlayer on $\mathrm{Cu}(001)$. Fig. 1 is a schematic representation of the system. The parameters for the two proposed configurations from Ref. 11 and 12, hereafter referred as "structure I" and "structure II", are summarized in Table \&. The second and lower Cu layers are assumed to occupy bulk-like positions. The presence of oxygen atoms and the missing rows reduce the symmetry of the system and create nonequivalent environments for $\mathrm{Cu}$ atoms belonging to the same layer. We take this into account by allowing atoms of the first three layers to be nonequivalent as needed. They are marked by different numbers in Fig. 1. Thus we build LIZ's around oxygen and nine nonequivalent $\mathrm{Cu}$ atoms within the top five layers. We take the sites of missing $\mathrm{Cu}$ to be occupied by vacancies represented by MT-spheres with zero core electron density and requiring an additional LIZ to be built around the vacancy. These LIZ's contained 62 to 91 atoms depending on the local configuration, and the requirement that the calculated characteristics of the bulk system closely match those obtained from other reliable methods in the literature.

\section{RESULTS AND DISCUSSION}

Adsorption of gases on surfaces causes both short- and long-range effects on the electronic structure of the system. The short-range effect originates from changes in the local environment of some surface atoms which lead to modifications of their chemical bonds. The 
long-range effect induced by the Coulomb interaction between the chemisorbed overlayer an the substrate, on the other hand, may cause differing shifts in the local potentials at atomic sites with differing environments. As a result, charge transfer may be induced and the electronic state hybridizations at these sites may be modified.

Here we examine in details both the short- and long-range effects of the chemisorption of an oxygen overlayer on $\mathrm{Cu}(001)$ with the view of understanding the driving forces responsible for the observed missing row reconstructed $(2 \sqrt{2} \times \sqrt{2}) R 45^{\circ}$ superstructure. We present first a comparative study of the short-range effect in structures I and II through examination of the local densities of electronic states at the oxygen and top $\mathrm{Cu}$ sites and their hybridizations. To gain further insight into the nature of the $\mathrm{O}-\mathrm{Cu}$ bonding and subtle differences emerging from structures I and II, we resort to an analysis of the projections of these densities of states on cubic harmonics. These short-range effects, however, are related to the long-range ones responsible for shifts in the local potentials at each site. We do this next through a systematic evaluation of the effects of the Coulomb interaction on local potentials. This is followed by an analysis of the charge transfer in the system. Finally, we comment on the relative stability of the $c(2 \times 2)$ phase, in view of the findings here for the $(2 \sqrt{2} \times \sqrt{2}) R 45^{\circ}$ phase.

\section{A. Local densities of the pO- and dCu-electronic states}

The calculated densities of the $p$-states of the oxygen and $d$-states of its nearest neighbors $\mathrm{Cu} 1, \mathrm{Cu} 2$ and $\mathrm{Cu} 3$ atoms for structures I and II are plotted in Fig. 2. The densities of the $d \mathrm{Cu} 4$ through $d \mathrm{Cu} 9$ states $\left(N_{d}^{C u}(E)\right)$ are shown in Fig. 3. Since the difference between $N_{d}^{C u}(E)$ of $\mathrm{Cu} 6$ and $\mathrm{Cu} 7$ is found to be negligible, only one is displayed. Figs. 2 and 3 indicate a dramatic difference in $N_{l}^{n}(E)$ for atomic sites with different environments. The $N_{d}^{C u}(E)$ of the $\mathrm{Cu} 6$ and $\mathrm{Cu} 8$ atoms belonging to the third and fourth layers, respectively, differ slightly from bulk values, while that of $\mathrm{Cu} 9$ coincides with that of the bulk. The $d \mathrm{Cu} 4-$ and $d \mathrm{Cu} 5$-states, on the other hand, consist of a pronounced narrow peak together with some low energy structure. Finally, the densities of the $p \mathrm{O}$-states and $d$-states of oxygen's nearest neighbors are found to be significantly split and structured. This splitting results in two substructures ("a" and "b" in Fig. 2), which have the same energetic position and similar shape for all spectra. Such a behavior clearly indicates a strong hybridization and covalent 
binding between the $p \mathrm{O}$-states and the $d$-states of the neighboring $\mathrm{Cu}$ atoms. A relatively high intensity of the low energy peaks "a" in $N_{d}^{C u 3}(E)$ suggests that the $d$ Cu3-states are more involved in the $p-d$ hybridization than the $d \mathrm{Cu} 1-$ and $d \mathrm{Cu} 2$-states. To understand the origin of this difference we should remember that the degree of hybridization of electronic states depends on their spatial and energetic overlap. Since, in the present structures, the

$\mathrm{O}-\mathrm{Cu} 3$ interatomic distance is longer than $\mathrm{O}-\mathrm{Cu} 1$ and $\mathrm{O}-\mathrm{Cu} 2$ ones1, 2 , the strong $p \mathrm{O}-\mathrm{Cu} 3$ hybridization does not result from the larger spatial overlap. The energetic overlap, on the other hand, can be quantitatively characterized within the multiple scattering theory by comparison of the single-site resonance energies $\left(E_{r e s}^{n}\right)$ for neighboring sites, which reflect energetics of the local potentials and primarily govern the energy of local subbands2 The calculated $E_{\text {res }}^{n}$ for structures I and II, in Table 【1, show substantial variation of $E_{\text {res }}^{n}$ from site to site. As we shall see in section $\mathrm{C}$, these variations are a result of the long-range interaction and charge transfer in the system. Since from Table [1], the energetic separation between the $p \mathrm{O}$ and $d \mathrm{Cu} 3$ resonances is smallest, we can conclude that the strong covalent $p \mathrm{O}-d \mathrm{Cu} 3$ binding is caused by a large energetic overlap of these states. Also, as $E_{\text {res }}^{C u 4}$ and $E_{\text {res }}^{C u 5}$ are much higher than the others in Table [1], there is only a small coupling of the $d \mathrm{Cu} 4$ - and $d \mathrm{Cu} 5$-states with those of their neighbors as indicated by the low intensity of the structures seen in the low energy region in Fig. 3.

Having established the strong coupling between the $p \mathrm{O}$ and $d \mathrm{Cu} 3$ electronic states, we proceed next with an analysis of the projections of the local densities of states on cubic harmonics $\left(N_{l m}^{n}(E)\right)$ which provide more detailed description of covalent binding in the system and help isolate the reasons for the a noticeable difference between the shape and splitting of for structures I and II.

\section{B. Projections of the densities of states on the cubic harmonics}

Since in the geometric structures under consideration, the local environment of the oxygen atoms have a reduced symmetry (see Fig. 4) because of the missing rows, the local electronic states are expected to be anisotropic. Therefore, to build a consistent picture of chemical binding in the system, it is important to analyze the projections of electronic state $N_{l m}^{n}(E)$ on the cubic harmonics. Plots in Fig. 5 of such projections of $p \mathrm{O}$ - and the five $d \mathrm{Cu} 3$-states, calculated for structure I, indicate that they differ dramatically from each other. The O- 
$p_{z}$ and $\mathrm{Cu} 3-d_{3 z^{2}-1}$ states are found to be strongly split. They form a common subband represented by peaks "a" and "b", which are separated by about $0.5 \mathrm{Ry}$. This strong splitting together with the involvement of major part of these states in hybridization (redistributed into "a" and "b" regions) are a clear signature of strong covalent binding. The O- $p_{x}$ and Cu3$d_{x z}$ as well as $\mathrm{O}-p_{y}$ and $\mathrm{Cu} 3-d_{y z}$ states also form common split subbands, but not as strongly as the $\mathrm{O}-p_{z}-\mathrm{Cu} 3-d_{3 z^{2}-1}$ states. The cause of this difference in the hybridization becomes clear through a comparison of the angular distribution of corresponding cubic harmonics, which shows that the O- $p_{x}-\mathrm{Cu} 3-d_{z x}$ and $\mathrm{O}-p_{y}-\mathrm{Cu} 3-d_{z y}$ spatial overlap is lower than that of $\mathrm{O}-p_{z}-\mathrm{Cu} 3-d_{3 z^{2}-1}$ (Fig. 5). The Cu3- $d_{x y}$ and $\mathrm{Cu} 3-d_{x^{2}-y^{2}}$ states cannot be coupled with the oxygen electronic states by symmetry. Instead they hybridize weakly with the electronic states of $\mathrm{Cu} 4$ and $\mathrm{Cu} 5$, as reflected by low intensity structure at high energies in Fig. 5. The weakness of this particular hybridization can be traced to the high energetic separation of the single-site resonances (see Table 【1]).

In Fig. 6, the corresponding splitting of the $\mathrm{O}-p_{z}-\mathrm{Cu} 3-d_{3 z^{2}-1}$ subband for structure II is found to be larger (5.7 Ry versus 5.0 Ry) than that for structure I, implying stronger covalent binding. Although for the O- $p_{x}-\mathrm{Cu} 3-d_{z x}$ and $\mathrm{O}-p_{y}-\mathrm{Cu} 3-d_{z y}$ subbands it is harder to quantify the splitting, Figs. 5 and 6 indicate that these subbands are also split stronger for structure II than for structure I. Again, these differences in splitting can be traced to the energetic separation between the $p \mathrm{O}$ and $d \mathrm{Cu} 3$ single-site resonances found for the two structures (see Table प1]).

The projections $N_{d m}^{C u}(E)$ calculated for the other nearest neighbors of oxygen, namely, $\mathrm{Cu} 1$ and $\mathrm{Cu} 2$ are shown in Fig. 7 and 8, respectively. Only a minor part of the $d \mathrm{Cu} 1-$ and $d \mathrm{Cu} 2$-electronic states are found to contribute to the peaks responsible for the $p \mathrm{O}-$ $d \mathrm{Cu}$ hybridization. As mentioned above, the single-site $d \mathrm{Cu} 1-$ and $d \mathrm{Cu}$ 2-resonances are energetically separated from the $p \mathrm{O}$-resonance that reduces the hybridization. The low energetic structures of $N_{d m}^{C u 1}(E)$ and $N_{d m}^{C u 2}(E)$ align with "a" peaks of $N_{p m}^{O}(E)$, which are formed with the $p \mathrm{O}-d \mathrm{Cu} 3$ hybridization. This suggests that the $d \mathrm{Cu} 1-$ and $d \mathrm{Cu} 2$-states just admix with the $p \mathrm{O}-d \mathrm{Cu} 3$-subband and the $\mathrm{O}-\mathrm{Cu} 1$ and $\mathrm{O}-\mathrm{Cu} 2$ covalent bonds are weaker than the O-Cu3 bond. 


\section{Long-range Coulomb interaction and charge transfer}

For a deeper understanding of the factors controlling the electronic and atomic structures of the $\mathrm{O} / \mathrm{Cu}(001)$ system, we turn now to considerations of long-range effect of the Coulomb interaction as expressed by the Madelung potential and the subsequent charge transfer in the surface region. To get a simple and intuitive picture we first calculate the Madelung potential for several surface structures of $\mathrm{Cu}(001)$ with $0.5 \mathrm{ML}$ of $\mathrm{O}$, within a simple "frozen" charge density approximation using Eq. 2. Namely, we calculate the effective charges $q_{i \alpha}$ introduced in Eq. 3 using bulk-like electronic density for $\mathrm{Cu}$ and atom-like density for $\mathrm{O}$. Such an approach gives us a pure geometric effect of the long-range interaction. As expected, the presence of the surface itself causes only small perturbation in the Madelung potential. This is represented by the low values in the column under "clean" $(\mathrm{Cu}(001)$ in Table III]. The admission of $0.5 \mathrm{ML}$ oxygen on bulk terminated $\mathrm{Cu}(001)$ causes large perturbation in the Madelung potential at atomic sites in the top few layers, as seen in the column denoted by BT in Table III. By removing every fourth row of $\mathrm{Cu}$ atom from this bulk terminated $\mathrm{O} / \mathrm{Cu}(001)$ system, we find the perturbation of the Madelung potential to be significantly reduced in the top $\mathrm{Cu}$ layer and slightly increased in some of the $\mathrm{Cu}$ sites below (see column labeled MR in Table [II]). The structures BT and MR are naturally artificial not only in terms of the positions of the top layer $\mathrm{Cu}$ atoms, but also that of the $\mathrm{O}$ which is taken to occupy the hollow sites in the surface plane. They have been chosen to provide benchmarks for the relative stability of the two observed $\mathrm{O}$ superstructures. The calculated perturbation

of the Madelung potentials for structure II, i.e. $(2 \sqrt{2} \times \sqrt{2}) R 45^{\circ} \mathrm{O}$ phase with $\mathrm{Cu}$ atoms in relaxed and reconstructed positions, in Table [II, indeed display significant reductions from the values of BT and MR configurations, for all atomic sites. Finally, in Table III we have also included the calculated quantities for the $c(2 \times 2)$ structure (parameters taken from Ref. 11 for a coverage less than 0.34 ML) for comparison. Clearly, the $c(2 \times 2)$ phase, albeit for $0.5 \mathrm{ML}$ coverage, invokes a much stronger perturbation of the Madelung potential than the one in structure II.

An electronic response to the strong perturbation of the Madelung potential is expected to be charge transfer in the vicinity of the surface. The resulting charge distribution would then determine the degree of ionicity of the system, and would influence single-site resonance energies, and consequently, the formation of covalent bonds. Charge transfer is thus a very 
important characteristic of systems. To characterize it quantitatively, the system has to be divided into spaces belonging to each atom and the charge within this space calculated. In the framework of the MT-approximation, non-overlapping spheres centered on the atomic sites can naturally represent these spaces. A complexity of the present system is that interatomic bond lengths for a number of atoms are different because of the differences in their local environment. Thus different volumes belong to different atomic sites. To make the site charges comparable, we have built the spheres with the fixed radius equal to one-half of the shortest interatomic distance $(1.807 \AA)$ and calculated the local valence charges by integrating the self-consistent valence electronic density over these spheres. The results in Table IV show a significant electronic charge transfer from $\mathrm{Cu}$ to $\mathrm{O}$ in both structures I and II. The charge transfer pattern is, however, interesting as $\mathrm{Cu} 2, \mathrm{Cu} 4$ and $\mathrm{Cu} 5$ donate part of their charge but $\mathrm{Cu} 1$ and $\mathrm{Cu} 3$ do not. These latter two nearest neighbors of $\mathrm{O}$, in fact, gain a small amount. Such a complex character of the charge transfer reflects the local potential perturbations caused by the missing $\mathrm{Cu}$ rows induced by the oxygen overlayer.

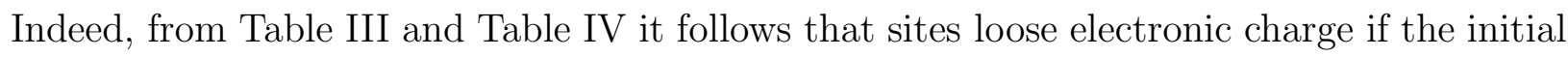
Madelung potential is higher and gain it if the potential is lower. Table $\mathbb{V}$ also reflects subtle differences in the charge transfer to the different sites in the two structures (I and II) considered here. Recall that covalent bonds have also been found stronger in structure II as compared to structure I.

Naturally the Coulomb interaction of the different sublattices plays a very important role in the above considerations. To get further insights into the difference arising from the two structural models chosen in this paper, we compare the Coulomb lattice energies $E_{\text {lat }}$ for these $\mathrm{O} / \mathrm{Cu}(001)$ phases. Within the MT approximation 2 $E_{\text {lat }}$ can be expressed as follows:

$$
E_{l a t}=\frac{1}{2} \sum_{i, \alpha} \sum_{j, \beta} M_{i \alpha, j \beta} q_{i \alpha} q_{j \beta}+\sum_{i, \alpha} \frac{1}{r_{M T}^{i \alpha}}\left(1.2\left(\omega_{i \alpha} \rho_{i}\right)^{2}+3 q_{i \alpha} \rho_{i} \omega_{i \alpha}\right),
$$

where $r_{M T}^{i \alpha}$ and $\omega_{i \alpha}$ are the MT-radii and MT-sphere volumes, respectively. The first sum of Eq. 4 represents the Madelung energy and the second comes from the charge neutrality condition. The $E_{l a t}$ thus includes the Coulomb interaction between the MT charges and that between them and the layer-dependent uniform interstitial charge density in the system. For comparative purposes it is sufficient to include the contribution of the oxygen overlayer and only the top five $\mathrm{Cu}$ layers to $E_{\text {lat }}$. Eq. 4 then yields values of -13.864Ry and -15.420Ry for $E_{l a t}$, per 2D unit cell, for structure I and structure II, respectively. The lower value of $E_{\text {lat }}$ 
along with the finding of stronger O-Cu covalent binding in structure II than in structure I, indicate preference for the former structure.

We have also calculated the Coulomb lattice energy for the $c(2 \times 2)$ O overlayer on $\mathrm{Cu}(001)$ using our self-consistent calculation results from Ref. 18 and obtained the value of $E_{l a t}=-$ 8.582Ry. This is much higher than that in the case of both structures I and II. This result is not surprising as we have already shown in Table $1 \mathrm{II}$ that the perturbation to the local Madelung potential at the atomic sites is also larger for the $c(2 \times 2)$ superstructure. Based on these findings we conclude that the long-range Coulomb interaction is the main factor which controls the phase formation in the $\mathrm{O} / \mathrm{Cu}(001)$ system and makes the $(2 \sqrt{2} \times \sqrt{2}) R 45^{\circ}$ phase preferable at oxygen coverage of $0.5 \mathrm{ML}$ in agreement with experimental findings.

\section{CONCLUSIONS}

The results presented above, provide the following microscopic picture for the formation of the $(2 \sqrt{2} \times \sqrt{2}) R 45^{\circ} \mathrm{O}$ overlayer on $\mathrm{Cu}(001)$. Oxygen adsorption in the four-fold hollow sites of $\mathrm{Cu}(001)$ induces a strong Coulomb perturbation on the top layer $\mathrm{Cu}$ sites. Missing row reconstruction of the $\mathrm{Cu}$ surface, lattice relaxation, and charge transfer help significantly reduce this perturbation. In response to the Coulomb perturbation, the electronic charge transfer from the host surface to $\mathrm{O}$ is found to have a complex character, involving two of the four nearest neighbors and two next nearest neighbors of O. The perturbation is screened partially and the self-consistent local potentials are such that the energetic separation between the single site $p \mathrm{O}$ - and $d \mathrm{Cu}$-resonances is different for different $\mathrm{Cu}$ sites. This energetic separation essentially determines the degree of the $p \mathrm{O}-d \mathrm{Cu}$ hybridization and the strengths of the covalent bonds formed between oxygen and its nearest neighbors. Thus, the $\mathrm{Cu} 3$ atom located right under $\mathrm{O}$ is involved in strong covalent binding with $\mathrm{O}$, whereas weak covalent bonds are formed between the $\mathrm{O}$ and its other nearest neighbors. The O$\mathrm{Cu} 3$ binding is found to be stronger in structure II than in structure I. Furthermore, as the Coulomb lattice energy is lower for the structure II than for structure I. In extending these consideration to the observed $c(2 \times 2)$ O overlayer on $\mathrm{Cu}(001)$ (for coverage less than $0.34 \mathrm{ML})$, we conclude that the $c(2 \times 2)$ structure is unstable, for half monolayer coverage, because it induces a strong perturbation at the surface that increases the Coulomb lattice energy of the system. Thus, according to our results, the long-range Coulomb interaction is 
the main driving force for the $\mathrm{O} / \mathrm{Cu}(001)$ surface atomic structure formation.

Finally, we note that adsorption processes on metal surfaces are extensively studied by means of such methods as Monte Carlo or molecular dynamics simulations. Many of them are based on the model potentials, which depend only on the local atomic environment, namely on the interatomic distance and the number of the nearest neighbors. Our findings indicate that an application of such approaches to $\mathrm{O} / \mathrm{Cu}(001)$ can hardly be successful. Indeed, according to our results, the long-range interaction in the system governs not only charge transfer (as usual), but also such "short-range" effects as the electronic state hybridization and covalent bond formation. Such an implicit impact cannot be taken into account even if a model potential somehow includes the long-range interaction along with a traditional short-range part. This suggests that a proper model potential for $\mathrm{O} / \mathrm{Cu}(001)$ can be built either by parameterization of the energetic surface obtained from first principle calculations or by development a sophisticated form, which takes into account the interplay between the long- and short-range parts.

\section{Acknowledgments}

This work was supported by the National Science Foundation (Grant No CHE9812397). The calculations were performed on the Origin 2000 supercomputer at the National Center for Supercomputing Applications, University of Illinois at Urbana-Campaign under Grant No DMR010001N. The work of TSR was also facilitated by the award of an Alexander von Humboldt Forschungspreis.

1 M. Kittel, M. Polcik, R. Terborg, J.-T. Hoeft, P. Baumgärtel, A. M. Bradshaw, R. L. Toomes, J.-H. Kang, D. P. Woodruff, M. Pascal, C. L. A. Lamont, and E. Rotenberg, Surf. Sci. 470, 311 (2001) and references therein.

2 S. Kono, S. M. Goldberg, N. F. T. Hall, and C. S. Fadley, Phys. Rev. Lett. 41, 1831 (1978).

3 P. Holland, B. J. Garrison, and N. Winograd, Phys. Rev. Lett. 43, 220 (1979).

4 U. Döbler, K. Baberschke, J. Stöhr, and D. A. Outka, Phys. Rev. B 31, 2532 (1985).

5 M. Sotto, Surf. Sci. 260, 235 (1992).

6 M. Wuttig, R. Franchy, and H. Ibach, Surf. Sci. 213, 103 (1989). 
7 J. G. Tobin, L. E. Klebanoff, D. H. Rosenblatt, R. F. Davis, E. Umbach, A. G. Baca, D. A. Shirley, Y. Huang, W. M. Kang, and S. Y. Tong, Phys. Rev. B 26, 7076 (1982).

8 T. Lederer, D. Arvanitis, G. Comelli, L. Tröger, and K. Baberschke, Phys. Rev. B 48, 15390 (1993).

9 T. Fujita, Y. Okawa, Y. Matsumoto, and K. Tanaka, Phys. Rev. B 54, 2167 (1996).

10 I. K. Robinson, E. Vlieg, and S. Ferrer, Phys. Rev. B 42, 6954 (1990).

11 A. Atrie, U. Bardi, G. Casalone, G. Rovida, E. Zanazzi, Vacuum 41, 333 (1990).

12 Ch. Wöll, R. J. Wilson, S. Chiang, H. C. Zeng, and K. A. R. Mitchell, Phis. Rev. B 42, 11926 (1990).

13 K. W. Jacobsen and J. K. Norskov Phys. Rev. Lett. 65, 1788 (1990).

14 E. A. Colbourn, J. E. Inglesfield Phys. Rev. Lett. 66, 2006 (1991).

15 P. V. Madhavan and M. D. Newton Chem. Phys. 86, 4030 (1987).

16 P. S. Bagus and F. Illas Phys. Rev. B 42, 10852 (1990).

17 T. Wiell, J. E. Klepeis, P. Bennich, O. Björnholm, N. Wassdahl, and A. Nilsson Phys. Rev. B 58, 1655 (1998).

18 S. Stolbov, A. Kara, and T. S. Rahman, cond-mat/0111145 (2001).

19 W. Kohn, L. J. Sham, Phys. Rev. 140, 1133 (1965).

20 Y. Wang, G. M. Stocks, W. A. Shelton, D. M. Nicholson, Z. Szotek, W. M. Temmerman, Phys. Rev. Lett. 75, 2867 (1995).

21 J. S. Faulkner and G. M. Stocks, Phys. Rev. B 21, 3222 (1980).

22 S.V. Stolbov, J. Phys.: Condensed Matter 94691 (1997)

23 S. Stolbov, M. Mironova, K. Salama, Supercond. Sci. Technol. 121071 (1999).

24 L.Szunyogh, B.Ujfalussy, P.Weinberger, J.Kollar, Phys.Rev. B 49, 2721 (1994).

25 O. Gunnarsson, B. I. Lundqvist, Phys. Rev. B 13, 4274 (1976).

26 P. Weinberger, Electron Scattering Theory for Ordered and Disordered Matter (Clarendon Press, Oxford, 1990).

27 P. C. Schmidt, Phys. Rev. B 315015 (1985). 


\section{Figure Captions}

Fig. 1. Schematic illustration of the $\mathrm{O} / \mathrm{Cu}(001)(2 \sqrt{2} \times \sqrt{2}) R 45^{\circ}$ structure.

Fig. 2. Densities of the $p$-electronic states of oxygen and $d$-electronic states of its nearest neighbors calculated for structure I (solid line) and structure II (dashed line).

Fig. 3. Densities of the $d$-electronic states of the $\mathrm{Cu} 4$ through $\mathrm{Cu} 9$ atoms calculated for structure I (solid line) and structure II (dashed line).

Fig. 4. Local environment of oxygen atom in the $(2 \sqrt{2} \times \sqrt{2}) R 45^{\circ} \mathrm{O} / \mathrm{Cu}(001)$ structure.

Fig. 5. Projections of the $p \mathrm{O}-$ and $d \mathrm{Cu} 3$-electronic states calculated for structure $\mathrm{I}$.

Fig. 6. Projections of the $p \mathrm{O}-$ and $d \mathrm{Cu} 3$-electronic states calculated for structure II.

Fig. 7. Projections of the $d \mathrm{Cu}$ 1-electronic states calculated for structure I (solid line) and the structure II (dashed line).

Fig. 8. Projections of the $d \mathrm{Cu} 2$-electronic states calculated for structure I (solid line) and structure II (dashed line). 
TABLE I: Structural parameters proposed from Ref.1 (structure I) and Ref.12 (structure II) which are used in our calculations

\begin{tabular}{ccc}
\hline \hline Parameter & Structure I & Structure II \\
\hline$z_{O}(\AA)$ & 2.05 & 2.14 \\
$z_{C u 1}(\AA)$ & 2.14 & 1.94 \\
$z_{C u 2}(\AA)$ & 1.88 & 2.04 \\
$\delta x_{C u 2}(\AA)^{a}$ & 0.29 & 0.30 \\
\hline \hline
\end{tabular}

${ }^{a}$ the $\mathrm{Cu} 2$ atom displacement along the $\mathrm{x}$ direction toward the missing row

TABLE II: Energetic positions (Ry) of the single site $p \mathrm{O}$ - and $d \mathrm{Cu}$-resonances $E_{r e s}^{n}$ counted from the MT-zero level

\begin{tabular}{ccc}
\hline \hline Site & Structure I & Structure II \\
\hline $\mathrm{O}$ & 0.232 & 0.222 \\
$\mathrm{Cu} 1$ & 0.354 & 0.377 \\
$\mathrm{Cu} 2$ & 0.416 & 0.447 \\
$\mathrm{Cu} 3$ & 0.316 & 0.283 \\
$\mathrm{Cu} 4$ & 0.492 & 0.493 \\
$\mathrm{Cu} 5$ & 0.533 & 0.516 \\
$\mathrm{Cu} 6$ & 0.403 & 0.408 \\
$\mathrm{Cu} 7$ & 0.408 & 0.417 \\
$\mathrm{Cu} 8$ & 0.382 & 0.388 \\
$\mathrm{Cu} 9$ & 0.382 & 0.382 \\
\hline \hline
\end{tabular}


TABLE III: The variation of the Madelung potential (in Ry) at different atomic sites with respect to the bulk value for the following configurations: clean $\mathrm{Cu}(001)$ surface (Clean); O on bulk terminated $\mathrm{Cu}(001)(\mathrm{BT})) ;(2 \sqrt{2} \times \sqrt{2}) R 45^{\circ} \mathrm{O}$ on bulk terminated $\mathrm{O} / \mathrm{Cu}(001)$ with missing rows $(\mathrm{MR}) ;$ structure II; $c(2 \times 2) \mathrm{O}$ on $\mathrm{Cu}(001)(c(2 \times 2))$

\begin{tabular}{ccccccc}
\hline \hline Layer & Site & Clean & BT & MR & Structure II & $c(2 \times 2)$ \\
\hline \multirow{2}{*}{ I } & $\mathrm{O}$ & - & -0.329 & -0.511 & -0.880 & -0.379 \\
& $\mathrm{Cu} 1$ & -0.007 & 1.438 & 0.495 & 0.219 & 1.810 \\
& $\mathrm{Cu} 2$ & -0.007 & 1.432 & 1.022 & 0.660 & 1.810 \\
\hline \multirow{2}{*}{$\mathrm{II}$} & $\mathrm{Cu} 3$ & 0.002 & 0.149 & -0.207 & 0.008 & -0.113 \\
& $\mathrm{Cu} 4$ & 0.002 & 0.285 & 0.271 & 0.299 & -0.113 \\
$\mathrm{III}$ & $\mathrm{Cu} 5$ & 0.002 & 0.149 & 0.478 & 0.162 & -0.113 \\
\hline $\mathrm{IV}$ & $\mathrm{Cu} 6$ & 0.001 & 0.020 & 0.016 & 0.011 & 0.01 \\
\hline $\mathrm{V}$ & $\mathrm{Cu} 7$ & 0.001 & 0.014 & 0.100 & 0.037 & 0.01 \\
\hline \hline
\end{tabular}

TABLE IV: Variations of the local valence electronic charge from the bulk-like charge for Cu sites and from the atomic-like charge for the $\mathrm{O}$ site.

\begin{tabular}{cccc}
\hline \hline Layer & Site & Structure I & Structure II \\
\hline \multirow{2}{*}{ I } & $\mathrm{O}$ & 0.518 & 0.508 \\
& $\mathrm{Cu} 1$ & 0.102 & 0.015 \\
& $\mathrm{Cu} 2$ & -0.131 & -0.287 \\
\hline $\mathrm{Cu} 3$ & 0.069 & 0.050 \\
$\mathrm{II}$ & $\mathrm{Cu} 4$ & -0.164 & -0.175 \\
& $\mathrm{Cu} 5$ & -0.263 & -0.361 \\
\hline $\mathrm{III}$ & $\mathrm{Cu} 6$ & 0.006 & -0.017 \\
& $\mathrm{Cu} 7$ & -0.005 & -0.016 \\
\hline $\mathrm{IV}$ & $\mathrm{Cu} 8$ & 0.013 & 0.014 \\
\hline $\mathrm{V}$ & $\mathrm{Cu} 9$ & 0.0 & 0.0 \\
\hline \hline
\end{tabular}




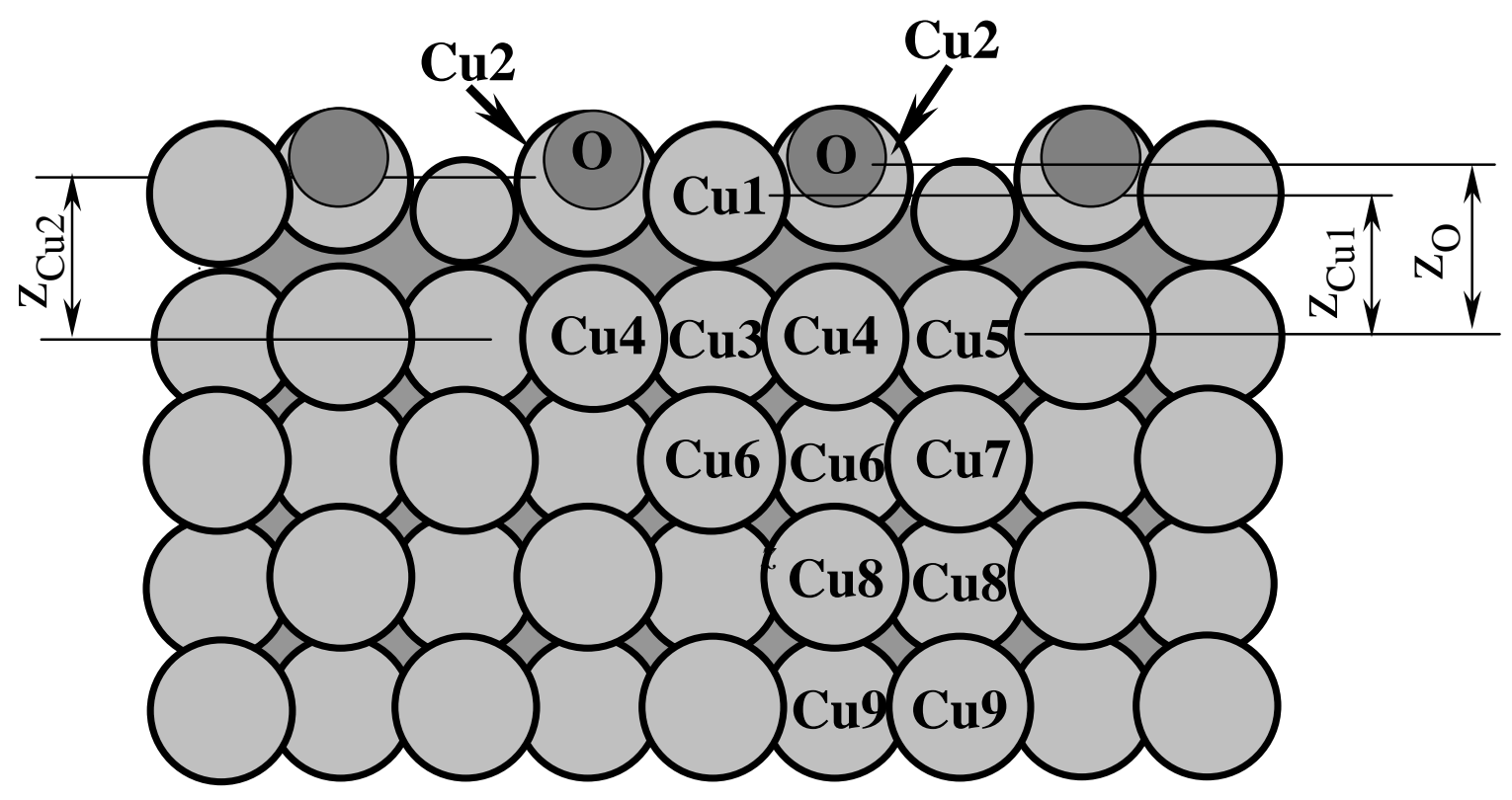




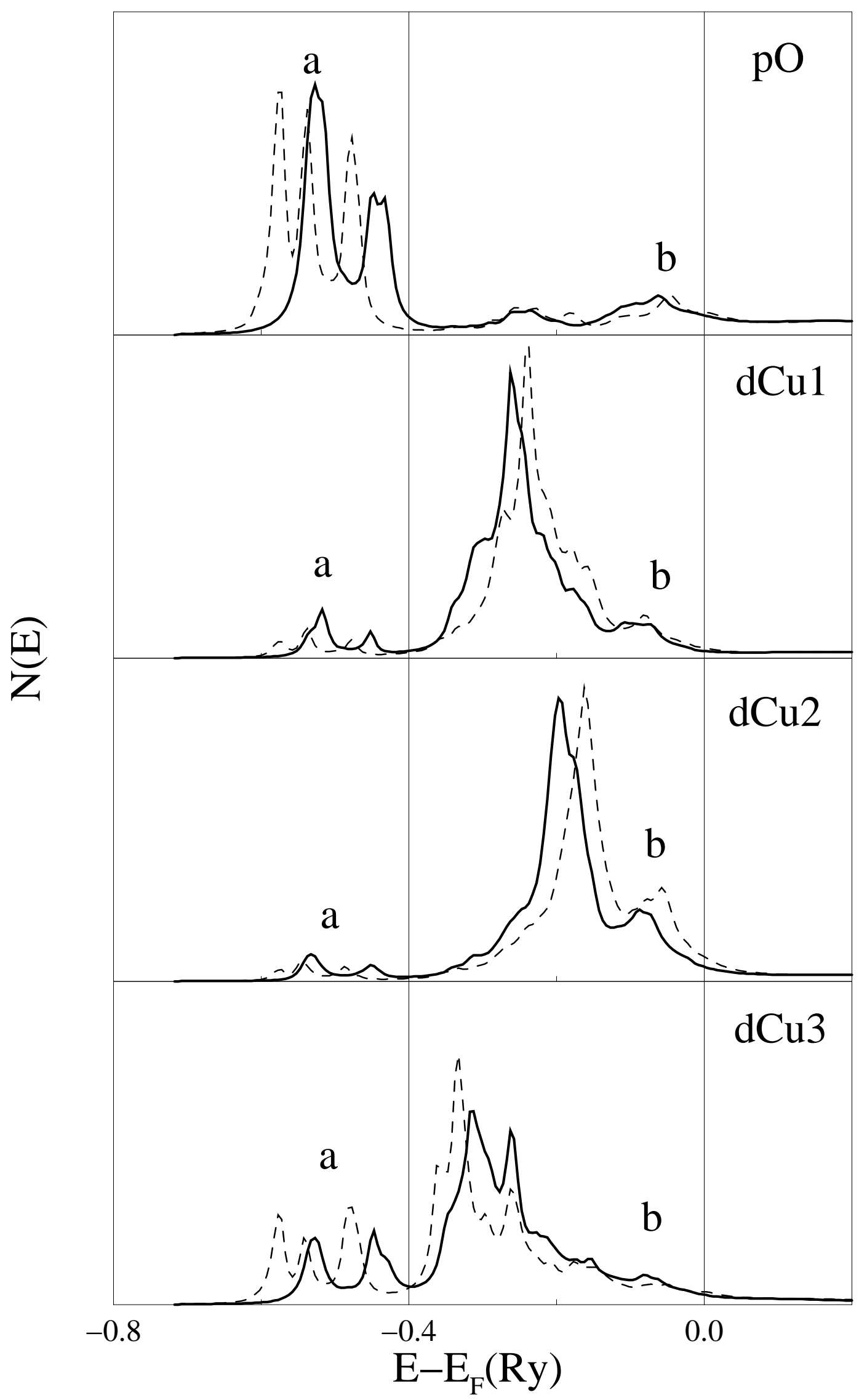




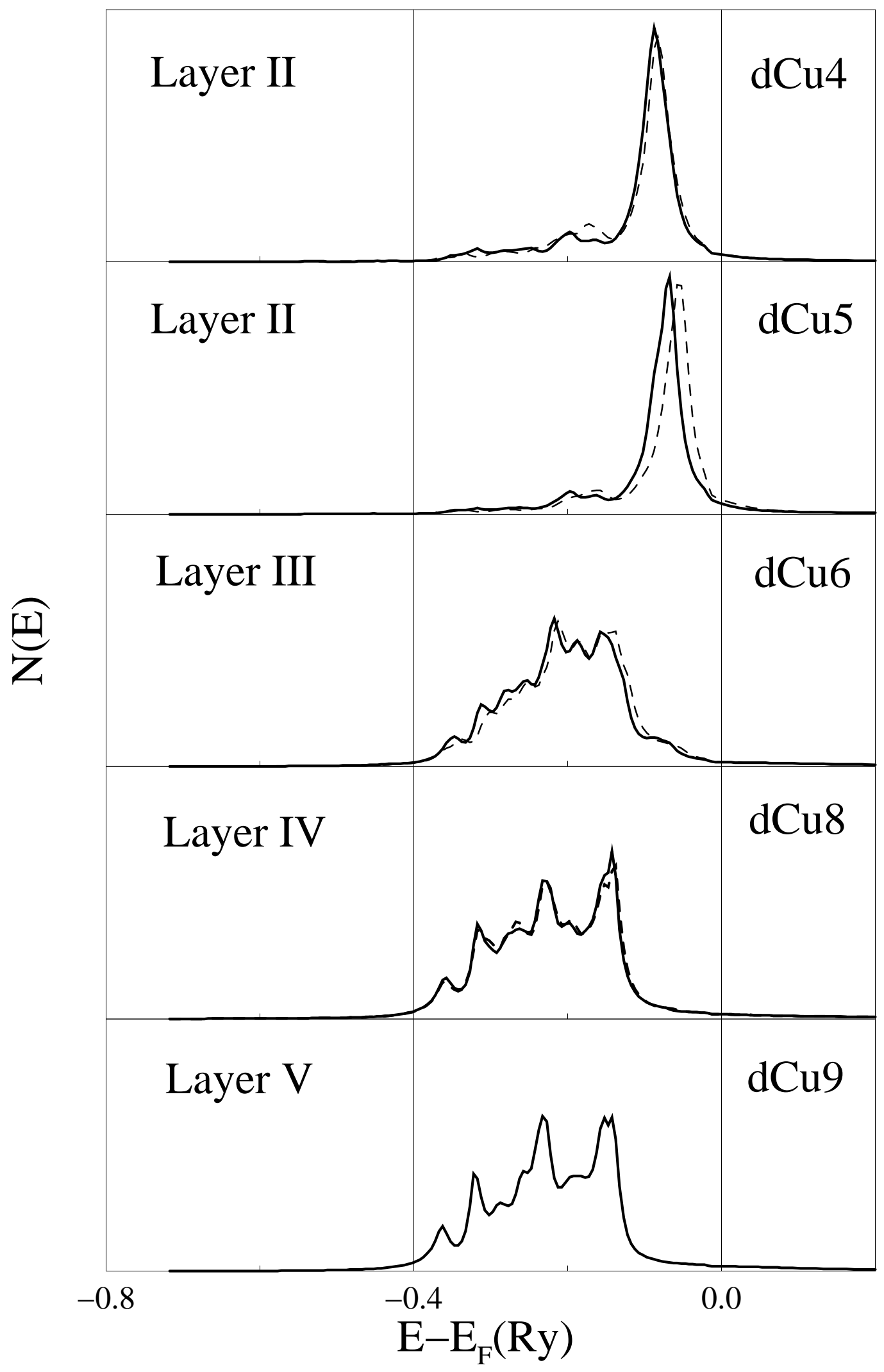




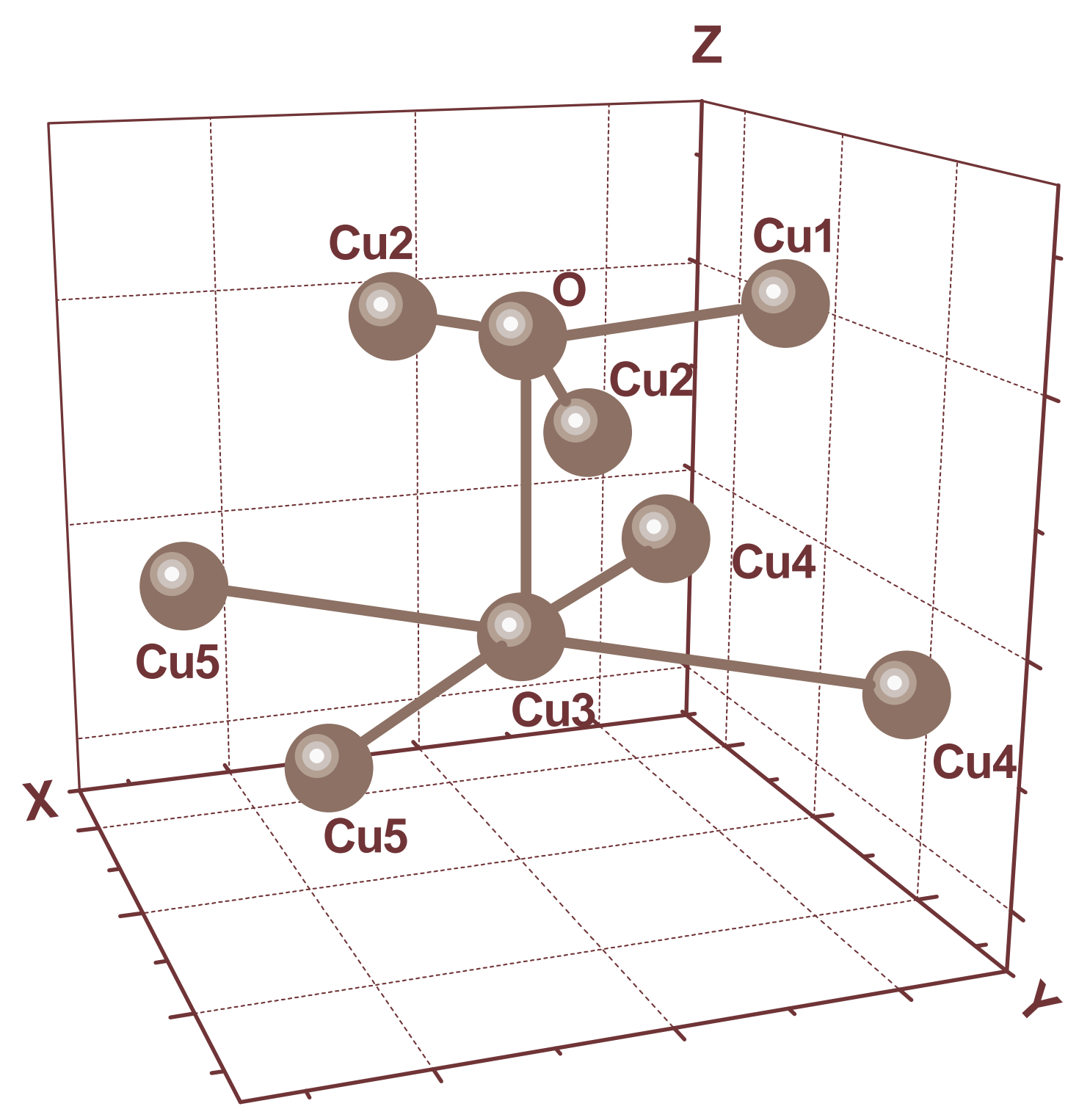




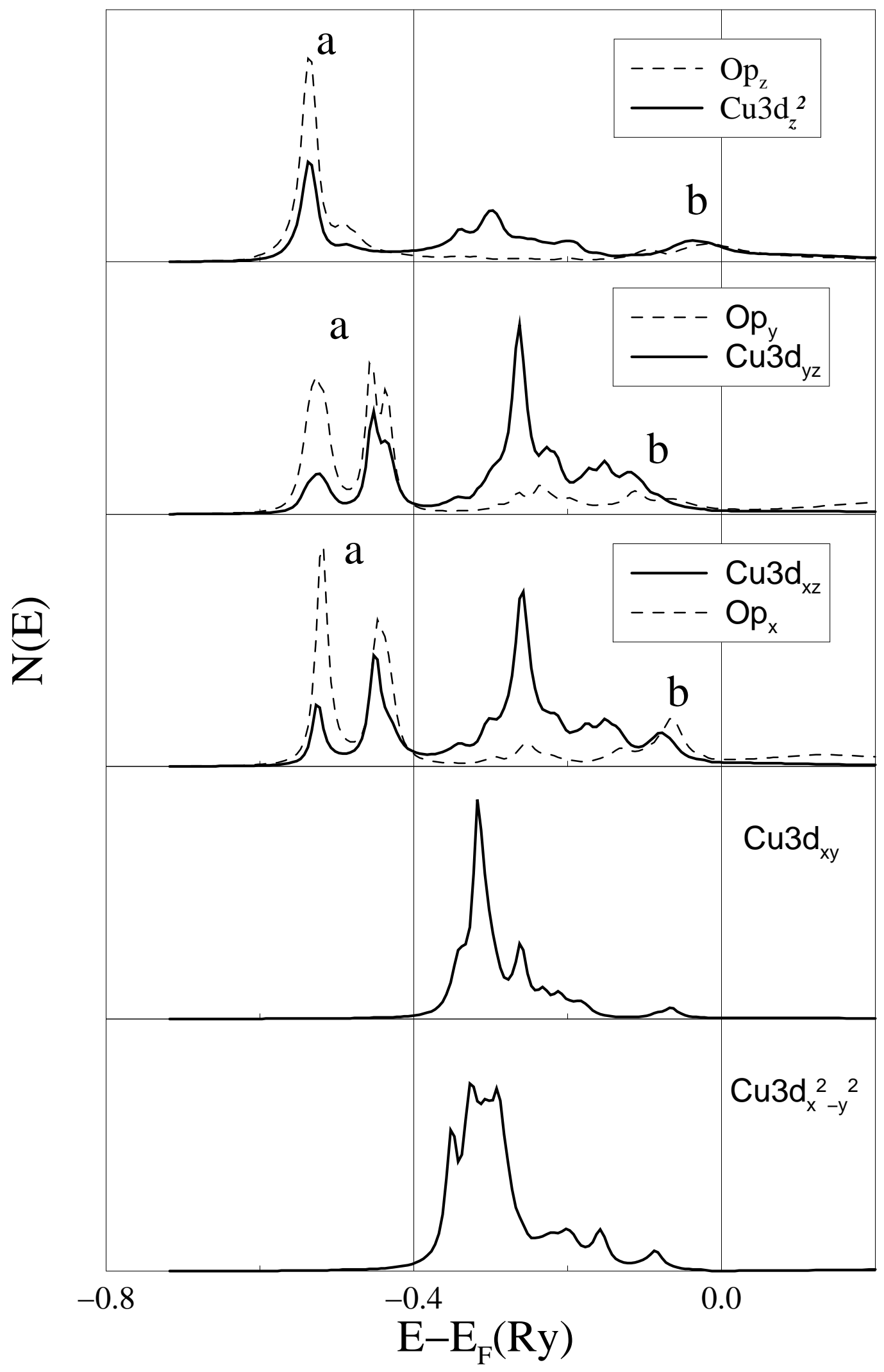




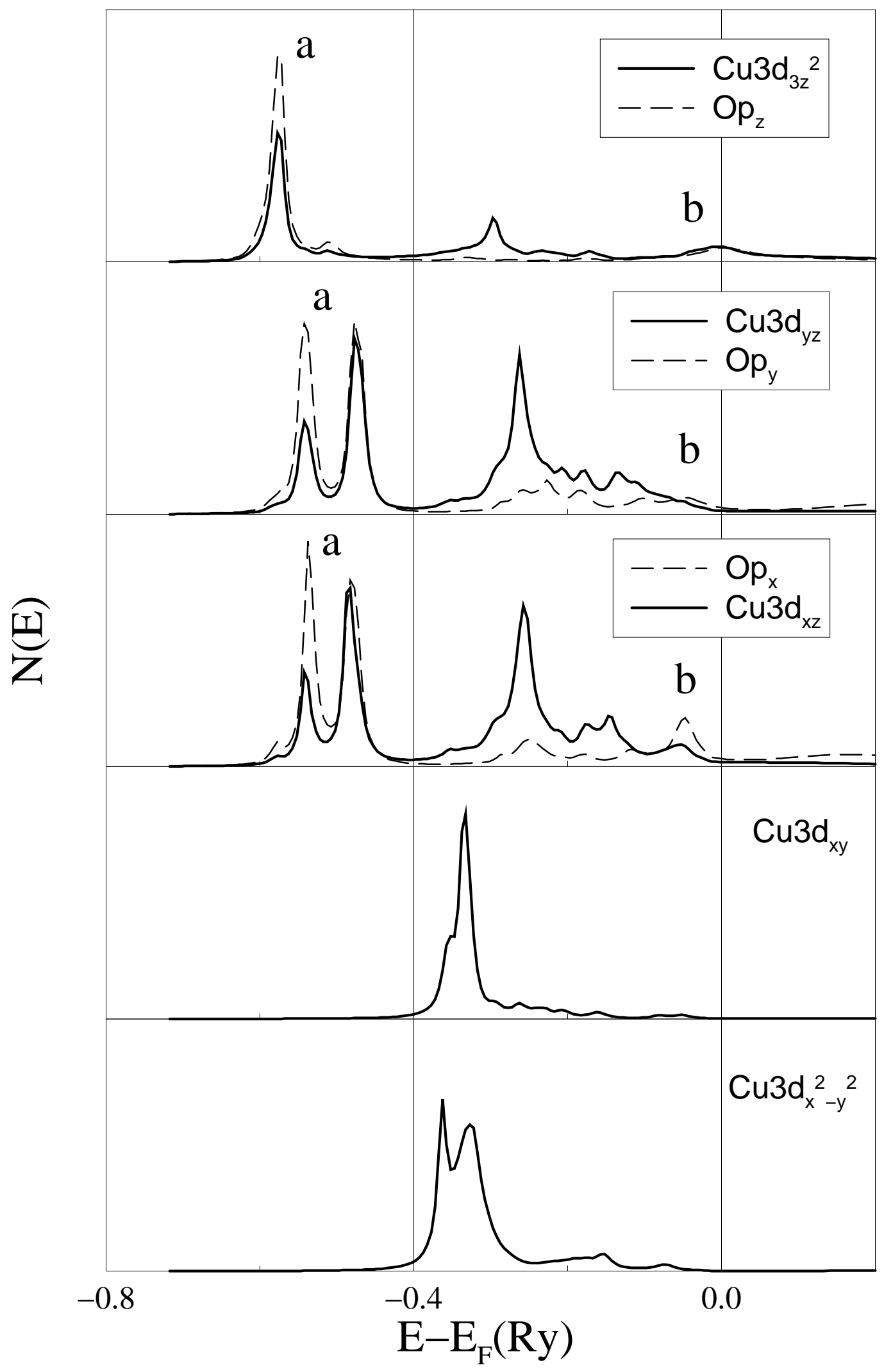




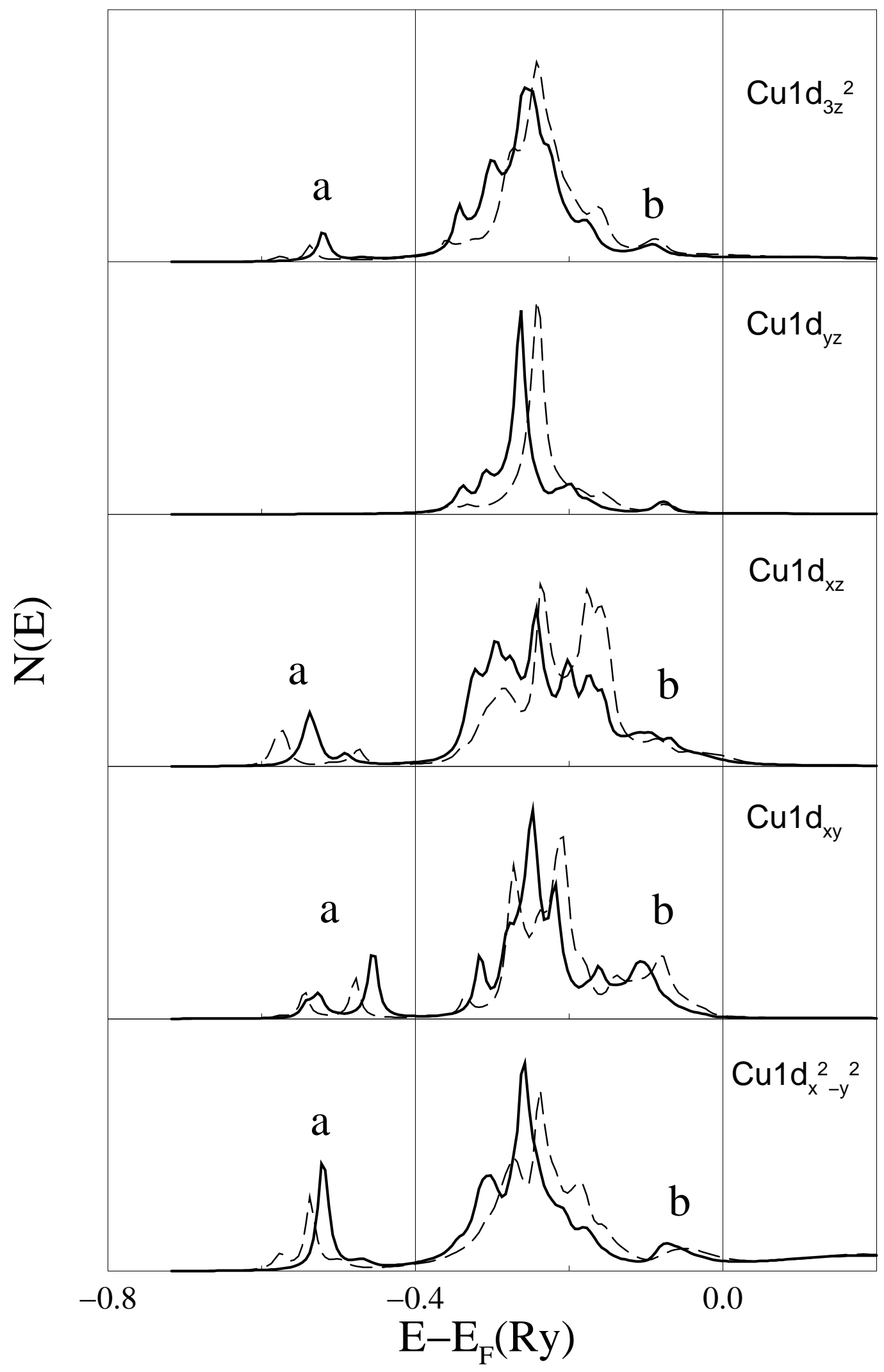




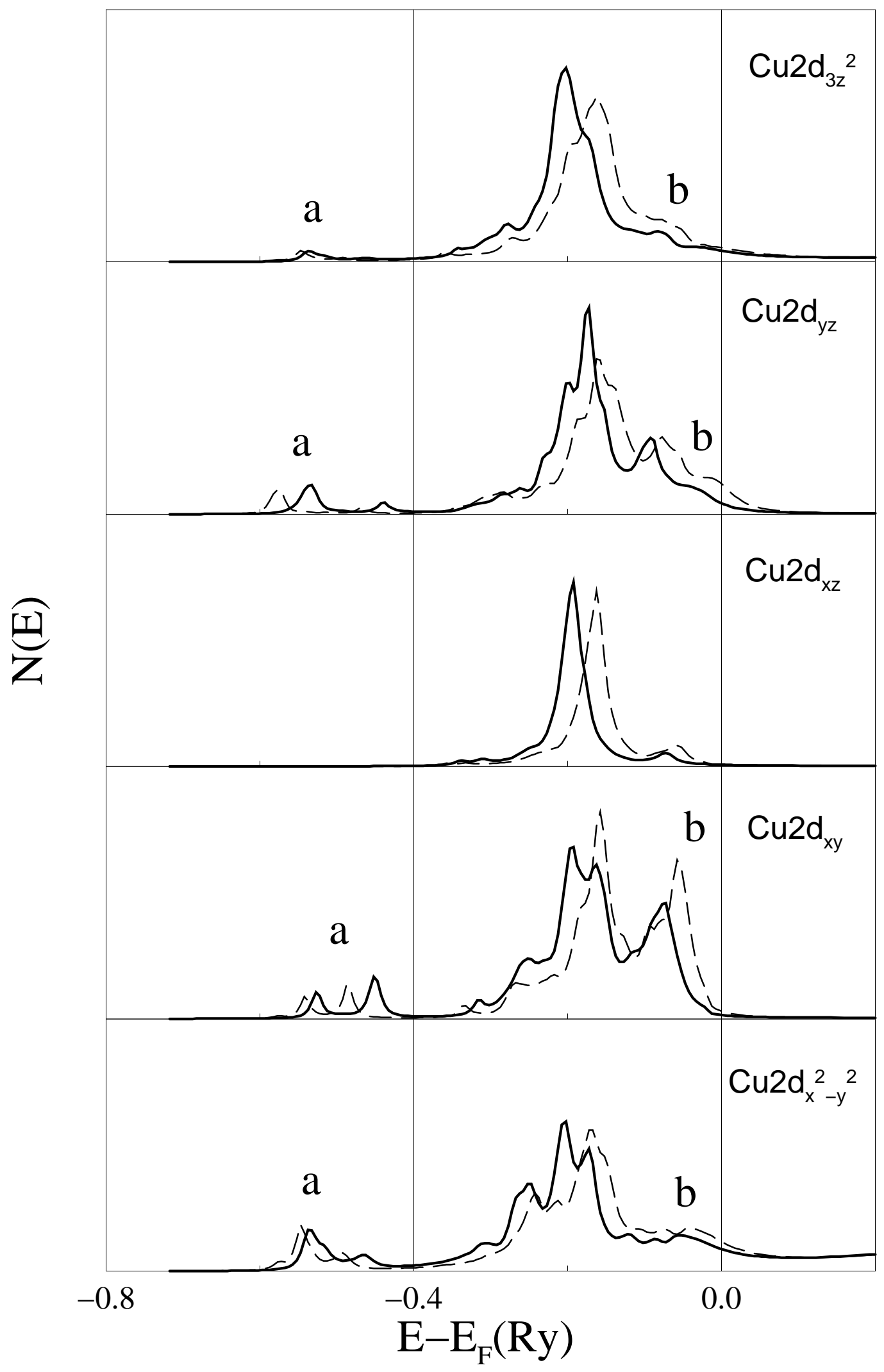

